\title{
Low-energy pion-nucleon scattering and the $\Delta$ resonance in lattice QCD
}

Constantia Alexandrou ${ }^{1,2, *}$, Giannis Koutsou ${ }^{1, * *}$, Stefan Krieg ${ }^{3,4, * *}$, Luka Leskovec ${ }^{5, * * *}$, Stefan Meinel ${ }^{6, \dagger}$, John W. Negele $^{7, \ddagger}$, Srijit Paul ${ }^{1,3,}$, Marcus Petschlies ${ }^{8,9, \pi}$, Andrew Pochinsky ${ }^{7, \|}$, Gumaro Rendon ${ }^{6, * *}$, Giorgio Silvi ${ }^{4, \dagger}$, and Sergey Syritsyn ${ }^{10,+\ddagger}$

${ }^{1}$ Computation-based Science and Technology Research Center, The Cyprus Institute

${ }^{2}$ Department of Physics, University of Cyprus, POB 20537, 1678 Nicosia, Cyprus

${ }^{3}$ Faculty of Mathematics und Natural Sciences, University of Wuppertal

${ }^{4}$ Institute for Advanced Simulation, Forschungszentrum Jülich GmbH, Jülich 52425, Germany

${ }^{5}$ Theory Center, Jefferson Lab, Newport News, VA 23606, USA

${ }^{6}$ Department of Physics, University of Arizona, Tucson, AZ 85721, USA

${ }^{7}$ Center for Theoretical Physics, Massachusetts Institute of Technology

${ }^{8}$ Helmholtz-Institut für Strahlen- und Kernphysik

${ }^{9}$ Bethe-Center for Theoretical Physics

${ }^{10}$ Department of Physics and Astronomy, Stony Brook University, Stony Brook, NY 11794, USA

\begin{abstract}
We report on our investigation of low-energy pion-nucleon scattering from lattice QCD with Wilsontype fermions. Our focus is on the isospin $I=3 / 2$ channel, which couples to the Delta resonance. We discuss our calculations aiming at the extraction of the Delta resonance mass, width and effective coupling at pion mass $250 \mathrm{MeV}$, using the Lüscher finite-volume method to extract the phase shift $\delta_{J=3 / 2, l=1}$.
\end{abstract}

\section{Introduction}

In this proceedings contribution we give an account of our extraction of low-energy pion-nucleon scattering properties in the channel $I\left(J^{P}\right)=\frac{3}{2}\left(\frac{3}{2}^{+}\right)$, which features the $\Delta$ resonance, from lattice QCD data at pion mass 250 $\mathrm{MeV}$. We will focus on the well-known parametrization in terms of Breit-Wigner parameters with resonance mass $m_{\Delta}$ and (energy-dependent) decay width $\Gamma_{\Delta-\pi N}(s)$. For the latter we will use the parametrization in leading-order effective theory of pions and nucleons, which defines the effective coupling $g_{\Delta-\pi N}$.

The $\Delta$ resonance is the lowest-lying meson-baryon resonance, which is accessible within purely elastic scattering, decaying with branching ratio approximately $100 \%$ to the QCD-stable ( = lattice QCD ground ) states of pion and nucleon. It is thus a natural starting point for a full-fledged scattering analysis based on Lüscher's finite-volume method [1], including its generalizations to moving frames, particles of non-equal mass, multiple

\footnotetext{
*e-mail: alexand@ucy.ac.cy

**e-mail: koutsou@cyi.ac.cy

***e-mail: s.krieg@fz-juelich.de

****e-mail: leskovec@jlab.org

†e-mail: smeinel@email.arizona.edu

†e-mail: negele@mit.edu

$\S$ e-mail: s.paul@hpc-leap.eu

II e-mail: marcus.petschlies@hiskp.uni-bonn.de

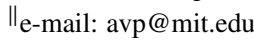

**e-mail: jgrs@email.arizona.edu

†† e-mail: g.silvi@fz-juelich.de

\$+e-mail: ssyritsyn@quark.phy.bnl.gov
}

channels [2-5] and extensions to particles of arbitrary spin $[6,7]$.

Even beyond in of itself the lattice QCD scattering analysis for $\Delta$ is of great interest for and a prequel to the calculation of pion-nucleon transition amplitudes via electro-weak currents, in the Delta resonance region. These include in particular the matrix elements for so-called $2 \rightarrow 1$ and $2 \rightarrow 2$ transitions as depicted diagrammatically in Fig. 1. Two very prominent examples

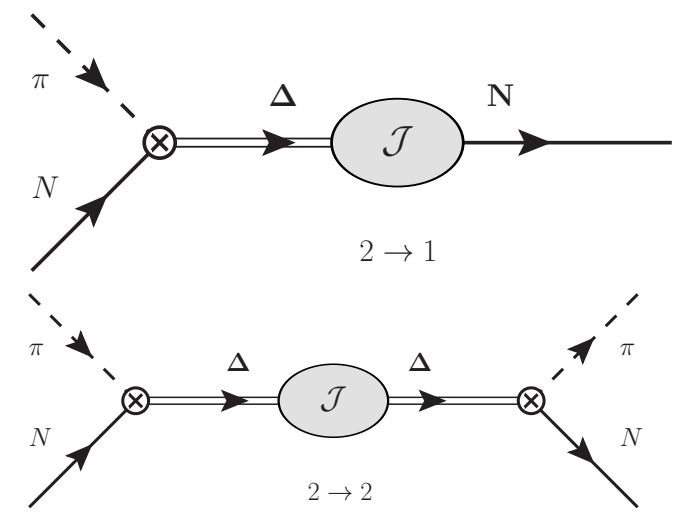

Figure 1. Diagrammatic illustration of $2 \rightarrow 1$ and $2 \rightarrow 2$ transition matrix elements of the $\Delta$

of foremost interest to us for the $2 \rightarrow 1$ case are the transition via the electromagnetic current $\mathcal{J}=V_{\mu}$ and the 
axial vector current $\mathcal{J}=A_{\mu}$. The former gives rise to the transition amplitude and thus cross-section for resonant pion-photoproduction off the nucleon, which can be compared directly to experiment. The lattice calculation of the radiative decay together with the strong decay, which is under consideration in this study, then gives in particular a complete ab-initio theory characterization of the decay structure of the $\Delta$ resonance.

The axial $N \rightarrow \Delta$ transition amplitude is important input to neutrino reaction cross sections like neutrino-nucleus scattering, which are investigated in contemporary experiments such as DUNE and MiniBooNE [8].

Common to all these analyses is the requirement to convert finite-volume lattice amplitudes to their infinite volume counterpart. This normalization based on the Lellouch-Lüscher factor [9] or its generalizations [10-14] in turn requires knowledge of the scattering matrix in the $\Delta$ resonance region, which is the goal our calculation presented here.

The pion-nucleon elastic scattering in the $\Delta$ channel has been the subject of several lattice QCD calculations. Among those are the analysis using the Michael/McNeile method [15, 16], requiring the lattice Hamiltonian to exhibit a $\Delta$-like state perturbatively close and below the pion-nucleon threshold and determining the resonance coupling based on the leading-order effective pion-nucleon interaction. The Lüscher method (to all orders in the effective $\pi N$ interaction) has been initially explored in several lattice QCD works [17-19]. However, the dedicated analysis of partial wave mixing is still to be achieved.

Here we discuss the first steps of our analysis of pionnucleon scattering in the $\Delta$ channel [20] from lattice QCD focusing on the irreps with leading $p$-wave contribution and neglecting partial wave mixing.

\section{Calculation of Lattice Correlation functions}

We consider QCD with up, down and strange quark on a Euclidean space-time lattice of spatial and temporal size $L=2.8 \mathrm{fm}$ and $T=2 L$. The gauge field ensemble features $N_{f}=2+1$ tree-level clover-improved Wilson quarks and the tree-level $O\left(a^{2}\right)$ Symanzik-improved gauge action [21] with two levels of HEX smearing of the gauge links coupling to the quarks.

For the specific ensemble A7 investigated here, the pion mass is $m_{\pi} \approx 258 \mathrm{MeV}$ and with an ensuing $m_{\pi} L \approx$ 3.6, residual exponential finite volume effects are expected to be sufficiently suppressed for the pertinent polynomial finite-volume effects underlying the Lüscher method to be dominant. For convenience all relevant ensemble parameters are collected in Table 1.

We perform the spectral analysis for the isospin channel $I=3 / 2, I_{3}=+3 / 2$ based on the generalized eigenvalue problem [22] for a matrix of correlation functions with single-hadron operators of $\Delta$-type and 2-hadron operators of $\pi N$ type. In detail, we list the operators in Eqs..

\begin{tabular}{|c|ccccc|}
\hline Label & $N_{s}^{3} \times N_{t}$ & $L(\mathrm{fm})$ & $a(\mathrm{fm})$ & $m_{\pi}(\mathrm{MeV})$ & $m_{\pi} L$ \\
\hline \hline $\mathrm{A} 7$ & $24^{3} \times 48$ & 2.8 & 0.116 & 258 & 3.6 \\
\hline
\end{tabular}

Table 1. Parameters of the ensemble A7 used in this work [].

(1) to (6) below.

For the single-hadron interpolators we use standard quark-antiquark and three-quark operators with two spinstructures for nucleon and Delta each.

$$
\begin{aligned}
\pi(t, \vec{p}) & =\sum_{\vec{x}} \bar{d}(t, \vec{x}) \gamma_{5} u(t, \vec{x}) e^{i \vec{p} \cdot \vec{x}} \\
N_{\mu}^{(1)}(t, \vec{p}) & =\sum_{\vec{x}} \epsilon_{a b c}\left(u_{a}(t, \vec{x})\right)_{\mu}\left(u_{b}^{T}(t, \vec{x}) C \gamma_{5} d_{c}(t, \vec{x})\right) e^{i \vec{p} \cdot \vec{x}} \\
N_{\mu}^{(2)}(t, \vec{p}) & =\sum_{\vec{x}} \epsilon_{a b c}\left(u_{a}(t, \vec{x})\right)_{\mu}\left(u_{b}^{T}(t, \vec{x}) C \gamma_{0} \gamma_{5} d_{c}(t, \vec{x})\right) e^{i \vec{p} \cdot \vec{x}} \\
\Delta_{\mu i}^{(1)}(t, \vec{p}) & =\sum_{\vec{x}} \epsilon_{a b c}\left(u_{a}(t, \vec{x})\right)_{\mu}\left(u_{b}^{T}(t, \vec{x}) C \gamma_{i} u_{c}(t, \vec{x})\right) e^{i \vec{p} \cdot \vec{x}} \\
\Delta_{\mu i}^{(2)}(t, \vec{p}) & =\sum_{\vec{x}} \epsilon_{a b c}\left(u_{a}(t, \vec{x})\right)_{\mu}\left(u_{b}^{T}(t, \vec{x}) C \gamma_{i} \gamma_{0} u_{c}(t, \vec{x})\right) e^{i \vec{p} \cdot \vec{x}}
\end{aligned}
$$

The two-hadron interpolators of $N \pi$-type are the product of the above pion and nucleon operators

$$
\begin{aligned}
(N \pi)^{(i)}\left(t, \vec{p}_{N}, \vec{p}_{\pi}\right) & =N^{(i)}\left(t, \vec{p}_{N}\right) \pi\left(t, \vec{p}_{\pi}\right), \\
\vec{p}_{N}+\vec{p}_{\pi} & =\vec{P}, i=1,2
\end{aligned}
$$

with specified total momentum $\vec{P}$. The individual hadron momenta range in $0 \leq|\vec{p}| /(2 \pi / L) \leq \sqrt{3}$.

From the operator basis in Eqs. (1) to (6) above we build all (cross-)correlation functions $\pi-\pi$ for the pion, $N^{(i)}-N^{(k)}$ for the nucleon and $\Delta^{(i)}-\Delta^{(k)},(N \pi)^{(i)}-\Delta^{(k)}$, $(N \pi)^{(i)}-(N \pi)^{(k)}$ for the Delta channel. The corresponding types of Wick contractions for the latter are depicted in the diagrams of Fig. 2, where each diagram-type stands as a placeholder for 2 to 4 distinct Wick contractions. To compute the diagrams we follow our "sequential + stochastic + smearing" method, previously successfully applied in the calculation of elastic $\pi \pi$ and $K \pi$ scattering amplitudes $[23,24]$ : contractions are based on point-to-all, sequential and stochastic timeslice-to-all propagators as well propagator products from usage of the one-end-trick. The propagators receive Wuppertal source and sink smearing [25] at each meson and baryon vertex, using the APE smeared [26] gauge field in the smearing operator.

\section{Lattice spectrum determination and Lüscher analysis}

The finite-volume and discretization of the lattice box lead to the breaking of the continuum $S U(2) \times$ parity rotation-reflection symmetry group down to $O_{h}^{D}$ (superscript $D$ denoting double cover) for a system of hadrons 


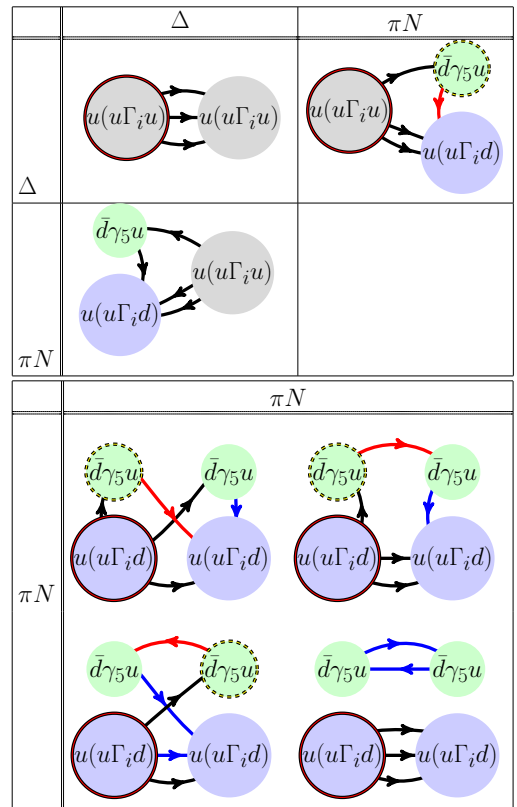

Figure 2. Topology-types of Wick contractions for $\Delta / N \pi$ correlation functions

\begin{tabular}{|l|l|l|l|}
\hline$\frac{L}{2 \pi} \vec{P}_{\text {ref }}\left[N_{\text {dir }}\right]$ & Group & $N_{\text {elem }}$ & $\Lambda(J): \pi\left(0^{-}\right)$ \\
\hline \hline$(0,0,0)[1]$ & $O_{h}^{D}$ & 96 & $A_{1 u}(0,4, \ldots)$ \\
$(0,0,1)[6]$ & $C_{4 v}^{D}$ & 16 & $A_{2}(0,1, \ldots)$ \\
$(0,1,1)[12]$ & $C_{2 v}^{D}$ & 8 & $A_{2}(0,1, \ldots)$ \\
$(1,1,1)[8]$ & $C_{3 v}^{D}$ & 12 & $A_{2}(0,1, \ldots)$ \\
\hline
\end{tabular}

Table 2. Little group, lattice irreps and angular momentum content per moving frame relevant for isospin $1 \pi$ operator.

\begin{tabular}{|l|l|l|l|}
\hline$\frac{L}{2 \pi} \vec{P}_{\text {ref } f}\left[N_{\text {dir }}\right]$ & Group & $N_{\text {elem }}$ & $\Lambda(J): N\left(\frac{1}{2}{ }^{+}\right)$ \\
\hline \hline$(0,0,0)[1]$ & $O_{h}^{D}$ & 96 & $G_{1 g}\left(\frac{1}{2}, \frac{7}{2}, \ldots\right) \oplus G_{1 u}\left(\frac{1}{2}, \frac{7}{2}, \ldots\right)$ \\
$(0,0,1)[6]$ & $C_{4 v}^{D}$ & 16 & $G_{1}\left(\frac{1}{2}, \frac{3}{2}, \ldots\right)$ \\
$(0,1,1)[12]$ & $C_{2 v}^{D}$ & 8 & $G\left(\frac{1}{2}, \frac{3}{2}, \ldots\right)$ \\
$(1,1,1)[8]$ & $C_{3 v}^{D}$ & 12 & $G\left(\frac{1}{2}, \frac{3}{2}, \ldots\right)$ \\
\hline
\end{tabular}

Table 3. Little group, lattice irreps and angular momentum content per moving frame relevant for isospin $1 / 2$ nucleon-type operators.

at rest. A boosted system with non-zero total momentum $\vec{P}$ will exhibit an even further reduced symmetry group depending on the boost vector $\vec{P}$, denoted as the little group $L G(\vec{P}) \subset O_{h}^{D}$. The interpolators in eqs.(1) to (6) are thus no longer classified according to continuum $S U(2)$ irreps of orbital and total angular momentum $l, J=l \oplus 1 / 2$, but according to the lattice irreducible representations (irreps) of $L G(\vec{P})$. In our analysis we consider the rest frame $\vec{P}=0$ as well the first three moving frames $\vec{P} /(2 \pi / L)=(0,0,1),(0,1,1),(1,1,1)$ (including permutations and sign changes for non-zero elements). The mixing pattern of the different $l$ and spin degrees of freedom for these frames under subduction to lattice irreps is shown in Tables 2 to 4 . To project our basis operators (or correlation functions) to the target lattice irreps, we use the

\begin{tabular}{|l|l|l|l|}
\hline$\frac{L}{2 \pi} \vec{P}_{r e f}\left[N_{\text {dir }}\right]$ & Group & $N_{\text {elem }}$ & $\Lambda(J): \Delta\left(\frac{3}{2}^{+}\right)$ \\
\hline \hline$(0,0,0)[1]$ & $O_{h}^{D}$ & 96 & $H_{g}\left(\frac{3}{2}, \frac{5}{2}, \ldots\right) \oplus H_{u}\left(\frac{3}{2}, \frac{5}{2}, \ldots\right)$ \\
$(0,0,1)[6]$ & $C_{4 v}^{D}$ & 16 & $G_{1}\left(\frac{1}{2}, \frac{3}{2}, \ldots\right) \oplus G_{2}\left(\frac{3}{2}, \frac{5}{2}, \ldots\right)$ \\
$(0,1,1)[12]$ & $C_{2 v}^{D}$ & 8 & $G\left(\frac{1}{2}, \frac{3}{2}, \ldots\right)$ \\
$(1,1,1)[8]$ & $C_{3 v}^{D}$ & 12 & $G\left(\frac{1}{2}, \frac{3}{2}, \ldots\right) \oplus F_{1}\left(\frac{3}{2}, \frac{5}{2}, \ldots\right) \oplus F_{2}\left(\frac{3}{2}, \frac{5}{2}, \ldots\right)$ \\
\hline
\end{tabular}

Table 4. Little group, lattice irreps and angular momentum content per moving frame relevant for isospin 3/2 $\Delta$-type operators.

group theory master formula

$$
O^{\Lambda, r, n}(t, \vec{p})=\frac{d_{\Lambda}}{g_{L G(\vec{P})}} \sum_{R \in L G(\vec{P})} \Gamma_{r, r}^{\Lambda}(R) U_{R} O(t, \vec{p}) U_{R}^{\dagger}
$$

In eq. (7) $O(\vec{p})$ stands for either single- or two-hadron operators (with appropriate 3-momentum arguments), $\Lambda$ denotes an irrep of $L G(\vec{P})$ with dimension $d_{\Lambda}, r \in\left\{1, \ldots, d_{\Lambda}\right\}$ one of its rows and $g$ gives the number of elements in the little group. $U_{R}$ is the unitary representation of the rotation on the operator algebra. For completeness, we list the action of rotations and reflections on the individual singlehadron interpolating fields for $\pi, N, \Delta$ in eqs. (8), (9) below.

$$
\begin{aligned}
U_{R} \pi(t, \vec{x}) U_{R}^{-1} & =\pi\left(t, R^{-1} \vec{x}\right) \\
U_{R} N(t, \vec{x}) U_{R}^{-1} & =S(R) N\left(t, R^{-1} \vec{x}\right) \\
U_{R} \Delta(t, \vec{x})_{k}^{\alpha} U_{R}^{-1} & =A(R)_{k k^{\prime}} S(R) \Delta_{k^{\prime}}\left(t, R^{-1} \vec{x}\right)
\end{aligned}
$$

$$
\begin{aligned}
I \pi(t, \vec{x}) I^{-1} & =-\pi(t,-\vec{x}) \\
I N(t, \vec{x}) I^{-1} & =\gamma_{t} N(t,-\vec{x}) \\
I \Delta(t, \vec{x}) I^{-1} & =\gamma_{t} \Delta(t,-\vec{x})
\end{aligned}
$$

In eq. (8) $S(R)$ is the 2-dimensional spin-1/2 representation of $S U(2)$ and $A(R)$ the 3-dimensional vector representation.

Given the many-to-one mapping of $S U(2)$ spin and vector degrees of freedom to lattice irrep states, an individual irrep $\Lambda$ can appear multiple times for a specific little group. The multiplicity is counted using the characters of the representation matrices [27, 28]

$$
m=\frac{1}{g_{L G(\vec{P})}} \sum_{R \in L G(\vec{P})} \chi^{\Gamma^{\Lambda}(R)} \chi^{M(R)},
$$

where $M$ denotes the spin-1/2 bispinor representation for the nucleon Dirac-spinor and the vector-bispinor product representation for the $\Delta$. The multiplicity pattern in the center-of-mass frame and the 3 moving frames used in this calculation is shown in the upper panel of Fig. 3 for the nucleon case and the lower panel for the $\Delta$.

From the set of irrep-projected single- and two-hadron interpolators with all included momentum configurations we draw a linearly independent set using the GramSchmidt procedure and thus arrive at a sum total of 1720 $\Delta$ - and $N \pi$ operators, across all frames and irreps, from 

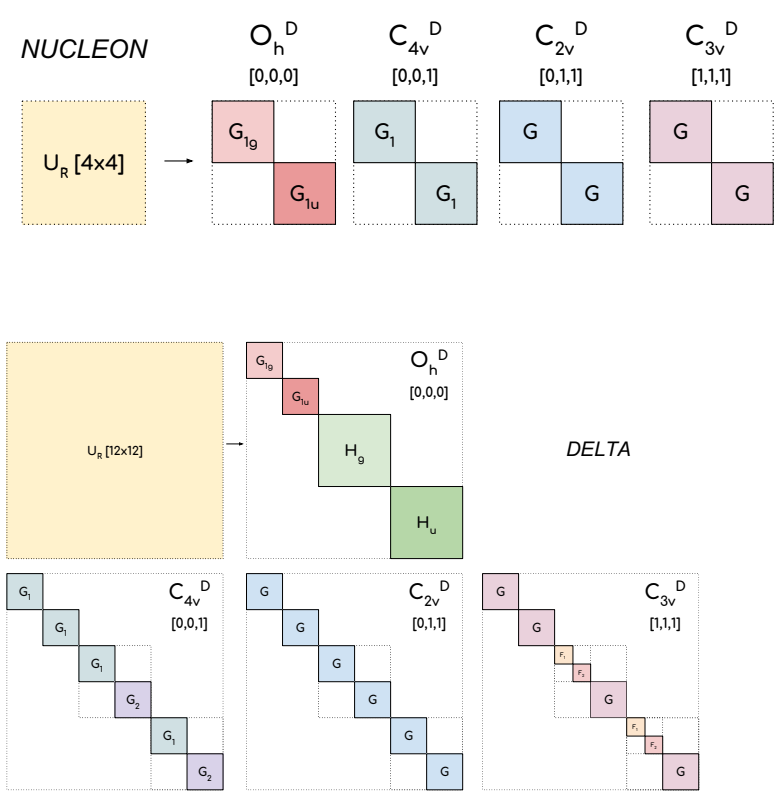

Figure 3. Multiplicities of irreps in frames with $\vec{P} /(2 \pi / L)=$ $(0,0,0),(0,0,1),(0,1,1),(1,1,1)$ for the nucleon (upper) and Delta.

which we then build the correlation matrices for the generalized eigenvalue problem. Total momentum $\vec{P}$ and the $L G(\vec{P})$ irrep are good quantum numbers for the finitevolume lattice, so the GEVP is solved per frame and per lattice irrep. In Fig. 4 we give two examples for the effective energy obtained from single exponential decay fits in the range $\left[t_{\min }, t_{\max }\right]$ to the generalized eigenvalues. In the top panel we show the effective energy plateau for the two lowest-lying levels of the combined $H_{g}$ and $H_{u}$ (timeforward and backward propagation) of the center-of-mass frame, $\vec{P}=0, L G(\vec{P})=O_{h}^{D}$. The bottom panel shows the same for the lowest 3 levels for the first moving frame and irrep $G_{1}$. The error bands with same color as the data points give the values from the nominal single exponential fits to the corresponding eigenvalues. The dashed lines give the $N \pi$ 2-particle elastic and the $N \pi \pi 3$-particle inelastic threshold. Moreover, the light green bars locate the non-interacting $N \pi$ energy levels to appreciate the finitevolume induced shift to the interacting levels.

In Fig. 5 we demonstrate the stability of fit results for the same levels as in Fig. 4 under variation of the fit range $\left[t_{\min }, t_{\max }\right]$ of the single exponential fits to the lowest-lying eigenvalues, in particular of the lower limit $t_{\min }$, which controls the impact of excited states. At each data point marking the fit result, the observed value for the fit's $\chi^{2} /$ dof is given. We find, that in general our extracted spectrum is stable within uncertainties for $t_{\min } / a \geq 4$.

\section{Scattering phase shift and resonance parameters}

At this stage in our analysis we disregard partial wave mixing and thus have a 1:1 relation between between the
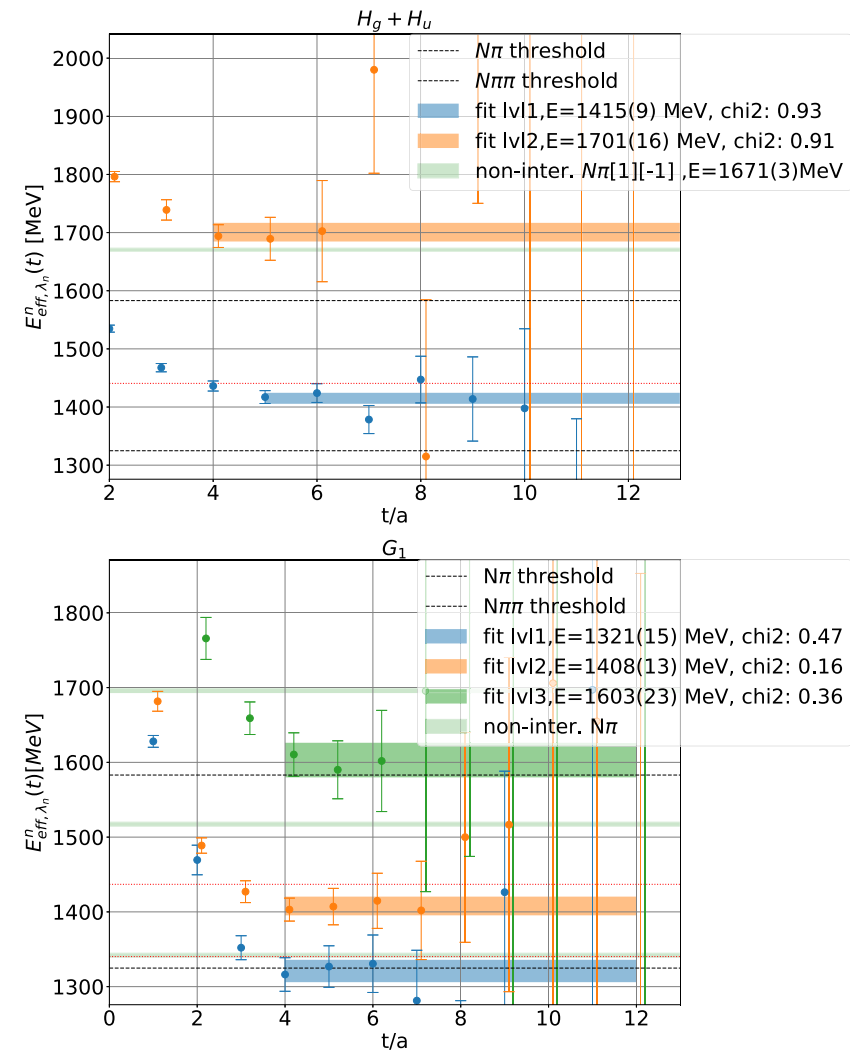

Figure 4. Examples of effective energy plateaus for combined $H_{u, g}$ in $\mathrm{cmf}$ and $G_{1}$ of the first moving frame.

lattice energy levels obtained in Sec. 3 and the infinite volume pion-nucleon $P$-wave elastic scattering phase shift $\delta_{J=3 / 2, l=1}$ from the Lüscher Quantization Condition (LQC) in eq. (11) [6].

$$
\operatorname{det}\left[M_{J l m, J^{\prime} l^{\prime} m^{\prime}}^{\vec{P}}(s)-\delta_{J J^{\prime}} \delta_{l l^{\prime}} \delta_{m m^{\prime}} \cot \left(\delta_{J l}(s)\right)\right]_{\sqrt{s}=E_{k}^{\Lambda, \vec{P}}}=0,
$$

to be fulfilled for the $k$ th energy level obtained from the frame with total momentum $\vec{P}$ and irrep $\Lambda$.

The LQC in eq. (11) is taken in the space of angular momentum states $|J, l, m\rangle$, for a given frame (total momentum $\vec{P})$. However, with irrep $\Lambda$, its row $r$ and occurrence $n$ good quantum numbers in the finite volume lattice calculation the Lüscher matrix can be block-diagonalized by a change to basis states $|\Lambda, r, n ; J, l\rangle[6,29]$. An example of the outcome of this transformation is depicted in Fig. 6 for the case of $\vec{P}=(0,0,1)(2 \pi / L)$ with $L G(\vec{P})=C_{4 v}^{D}$. Thus we obtain a corresponding factorized quantization condition, where individual factors solve the quantization of energy levels for individual irreps,

$$
\operatorname{det}\left[M_{J l n, J^{\prime} l^{\prime} n^{\prime}}^{\Lambda, \vec{P}}(s)-\delta_{J J^{\prime}} \delta_{l l^{\prime}} \delta_{m m^{\prime}} \cot \left(\delta_{J l}(s)\right)\right]_{\sqrt{s}=E_{k}^{\Lambda, \vec{P}}}=0 .
$$

To extract resonance parameters for the $\Delta$ we use the Breit-Wigner parametrization of the scattering amplitude 


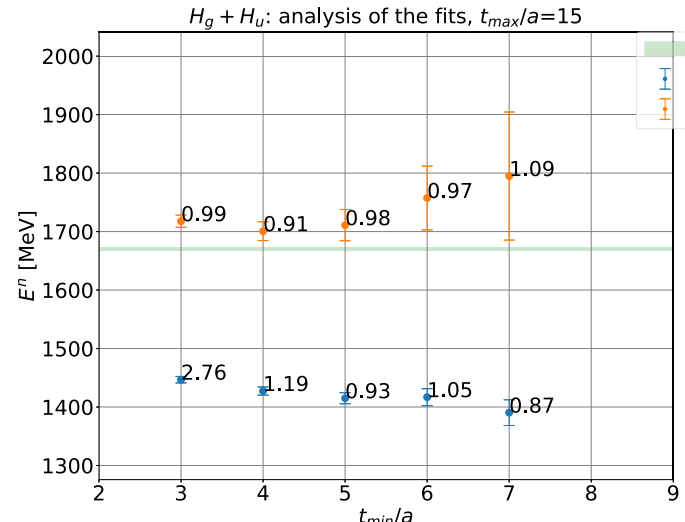

non-inter. $N \pi$

Fit IVI 1

Fit IvI 2

$G_{1}$ : analysis of the fits. $t_{\max } / a=12$

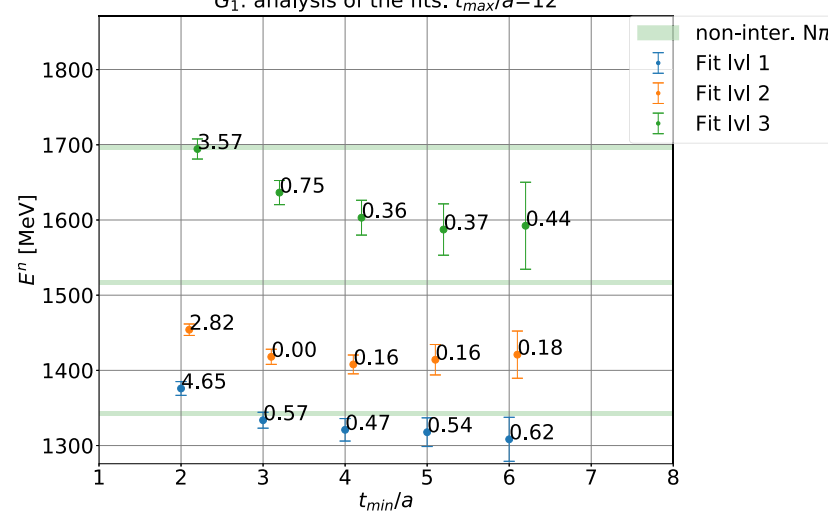

Figure 5. Check of stability of the GEVP energy levels by variation of lower end of the single exponential fit range; top: $\mathrm{cmf}$ first two levels; bottom: first three levels in $G_{1}$ of the first moving frame.

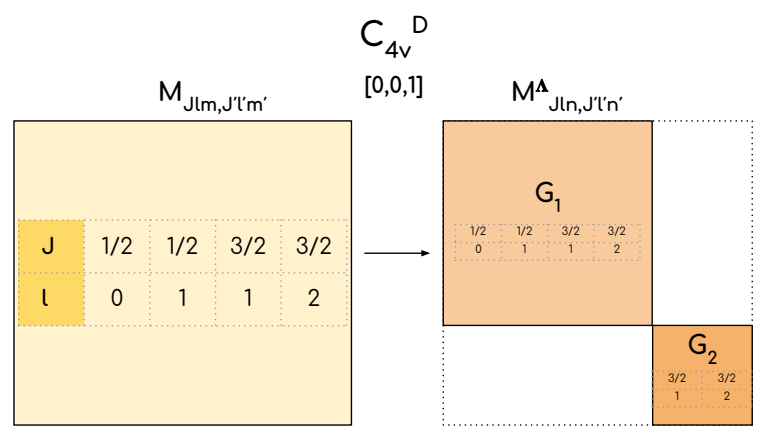

Figure 6. Block-diagonalization of the Lüscher matrix by change to irrep basis

(with contribution from a single partial wave at this point)

$$
t_{l}(s)=\frac{\sqrt{s} \Gamma(s)}{m_{R}^{2}-s-i \sqrt{s} \Gamma(s)},
$$

which incorporates the pole for the isolated, narrow Delta resonance.

Moreover, the $s$-dependence of the decay width $\Gamma$ is described using the result from leading-order effective theory of the pion-nucleon system [30], which introduces the

\begin{tabular}{|c|c|c|}
\hline Particle & $J^{P}$ & $\Gamma_{N \pi}[\mathrm{MeV}]$ \\
\hline \hline$\Delta(1232)$ & $3 / 2^{+}$ & $112.4(5)$ \\
\hline$\Delta(1600)$ & $3 / 2^{+}$ & $18(4)$ \\
$\Delta(1620)$ & $1 / 2^{-}$ & $37(2)$ \\
$\Delta(1700)$ & $3 / 2^{-}$ & $36(2)$ \\
$\ldots$ & $\ldots$ & $\ldots$ \\
\hline
\end{tabular}

Table 5. Experimentally observed resonances in the isospin-3/2 channel.

effective coupling $g_{\Delta-\pi N}$

$$
\Gamma_{E F T}^{L O}(s)=\frac{g_{\Delta-\pi N}^{2}}{48 \pi} \frac{E_{N}+m_{N}}{E_{N}+E_{\pi}} \frac{p^{* 3}}{m_{N}^{2}}
$$

To extract the target quantities $m_{R}$ and $g_{\Delta-\pi N}$ we employ the inverse Lüscher method: with the Breit-Wigner parametrization of the scattering amplitude in Eq. (13) and together with the energy dependence of the decay width in Eq. (14) the quantization condition in Eq. (12) allows for the prediction of the expected lattice energy levels (disregarding the effect of non-zero lattice spacing artefacts). We compare the predicted values ("model") with our observed energy levels ("avg") and fit the resonance parameters to minimize the cost function in eq. (15).

$$
\begin{aligned}
& \chi^{2}=\sum_{\vec{P}, \Lambda, n} \sum_{\vec{P}^{\prime}, \Lambda^{\prime}, n^{\prime}}\left(\sqrt{s_{n}^{\Lambda, \vec{P}}}[\text { avg }]-\sqrt{s_{n}^{\Lambda, \vec{P}}}[\text { model }]\right) \times \\
& \times\left[C^{-1}\right]_{\vec{P}, \Lambda, n}^{\vec{P}^{\prime}, \Lambda^{\prime}, n^{\prime}}\left(\sqrt{s_{n^{\prime}}^{\Lambda^{\prime}, \vec{P}^{\prime}}[\text { avg }]}-\sqrt{s_{n^{\prime}}^{\Lambda^{\prime} \vec{P}^{\prime}}}[\text { model }]\right)
\end{aligned}
$$

From the GEVP analysis in the nucleon channel we obtain our estimate for the nucleon mass given in Eq. (16) together with the pion mass for the ensemble studied here.

$$
m_{\pi}=258(1.1) \mathrm{MeV}, \quad m_{N}=1066.4(2.7) \mathrm{MeV}
$$

Our preliminary estimate of the Delta resonance mass and effective coupling to the pion-nucleon state is based on the evaluation of data from three frames and one irrep each, $\vec{P}=0, H_{g / u}, \vec{P}=(0,0,1) 2 \pi / L, G_{2}$ and $\vec{P}=$ $(0,1,1) 2 \pi / L, G$ (cf. Tab. 4). The first two cases have our target continuum partial wave $J=3 / 2$ as the leading contribution (with contamination from only higher partial waves, $J \geq 5 / 2$ ). The latter case on the other hand, has a contribution from $J=1 / 2$. However, considering the experimentally observed excitations [31] collected in Tab. 5 (at physical pion mass), we expect the dominant resonant features to be only mildly influenced by higher-lying resonance contributions, largely to the upper end of the energy spectrum considered in our study. From the ensuing fit we then obtain the parameters

$$
m_{\Delta}=1414(128) \mathrm{MeV}, \quad g_{\Delta-\pi N}=26(4)
$$

with fit quality $\chi^{2} /$ dof $=3.15$.

\section{Summary and outlook}

We present our data points for energy levels and phase shift $\delta_{J=3 / 2, l=1}$, which enter our fit, in Fig. 7. The green 


\begin{tabular}{lll}
\hline Collaboration & Ref. & Methodology \\
\hline Verduci (2014) & {$[18]$} & Dist., Lüscher \\
Alexandrou et.al. (2013) & {$[15]$} & Michael \& McNeile \\
Alexandrou et.al. (2015) & {$[16]$} & Michael \& McNeile \\
Andersen et.al. (2017) & {$[19]$} & Stoch. Dist., Lüscher \\
Our study (preliminary) & {$[20]$} & Smeared src., Lüscher \\
\hline Physical value (PDG) & {$[32]$} & pheno, K-matrix \\
\hline
\end{tabular}

Table 6. Collection of contemporary results of studies of the Delta resonance mass and $g_{\Delta-\pi N}$ in lattice QCD and from analysis of experimental data (bottom line): analysis methods used.

line shows the evaluation of the fit function with the best fit parameters and the shaded region gives the uncertainty obtained from the error propagation with the variancecovariance matrix of the fit parameters. We find in our

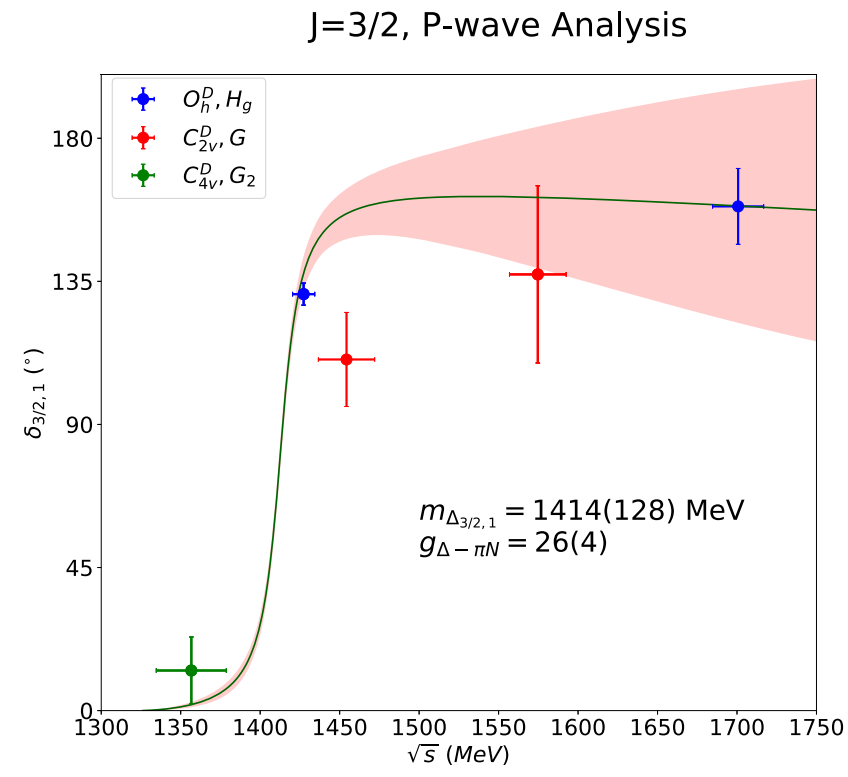

Figure 7. Our current result for the $I\left(J^{P}\right)=\frac{3}{2}\left(\frac{3}{2}^{+}\right)$phase shift using the spectrum from little groups $O_{h}^{D}, C_{4 v}^{D}$ and $C_{2 v}^{D}$.

data the generically expected features of resonant behavior in the phase shift with a steep rise from $\delta_{3 / 2,1}=0$ to $\approx 180$ degrees. However, at present the levels from $C_{2 v}^{D} / G$ give rise to some tension with the model prediction, leading to a $\chi^{2} /$ dof-value of about 3 in our fit. Their role deserves further investigation, in particular since right for this case the angular momentum content of the irrep starts at $J=1 / 2$.

Finally, we compare the preliminary results of our study presented here with other determinations of Delta resonance parameters in lattice QCD calculations in Tabs. 6, 7. The comparison can only be a superficial one at this point, as the results listed stem from very different calculations, without commonalities like pion mass or a continuum extrapolation. Nevertheless, we find rough agreement of $m_{\Delta}$ for comparable pion mass. The effective coupling is already in the region of the value extracted from phenomenological and K-matrix analysis of experimental data (last line in both tables). The latter

\begin{tabular}{llll}
\hline Ref. & $m_{\pi}[\mathrm{MeV}]$ & $m_{\Delta}[\mathrm{MeV}]$ & $g_{\Delta-\pi N}$ \\
\hline$[18]$ & 266 & $1396(19)$ & $19.90(83)$ \\
{$[15]$} & 360 & $1535(25)$ & $26.7(1.5)$ \\
{$[16]$} & 180 & $1350(50)$ & $23.7(1.3)$ \\
{$[19]$} & 280 & $1344(20)$ & $37.1(9.2)$ \\
{$[20]$} & 258 & $1414(128)$ & $26(4)$ \\
\hline$[32]$ & 140 & $1232(1)$ & $29.4(3), 28.6(3)$ \\
\hline
\end{tabular}

Table 7. Collection of contemporary results of studies of the Delta resonance mass and $g_{\Delta-\pi N}$ : numerical values obtained for the analyses listed in Tab. 6.

tendency is consistent for the dimensionless quantity $g_{\Delta-\pi N}$, which is expected to display only a mild quark mass dependence.

At the time of this report, we continue to build up the gauge statistics for the ensemble A7, which is the basis for the data shown here. Moreover, two immediate followup simulations at same pion mass but larger volume and at smaller pion mass of approximately $200 \mathrm{MeV}$ are in preparation.

\section{Acknowledgements}

This research used resources of the National Energy Research Scientific Computing Center (NERSC), a U.S. Department of Energy Office of Science User Facility operated under Contract No. DE-AC02-05CH11231. SM and GR are supported by the U.S. Department of Energy, Office of Science, Office of High Energy Physics under Award Number DE-SC0009913. SK is supported by the Deutsche Forschungsgemeinschaft grant SFB-TRR 55. SK and GS were partially funded by the IVF of the HGF. SM and SS also thank the RIKEN BNL Research Center for support. JN was supported in part by the DOE Office of Nuclear Physics under grant DE-SC-0011090. AP was supported in part by the U.S. Department of Energy Office of Nuclear Physics under grant DE-FC02-06ER41444. LL was supported by the U.S. Department of Energy, Office of Science, Office of Nuclear Physics under contract DE-AC05-06OR23177. SP was supported by the Horizon 2020 of the European Commission research and innovation programme under the Marie Sklodowska-Curie grant agreement No. 642069. MP gratefully acknowledges support by the Sino-German collaborative research center CRC-110. We acknowledge the use of the USQCD software QLUA [33] for the calculation of the correlators.

\section{References}

[1] M. Lüscher, U. Wolff, Nuclear Physics B 339, 222 (1990)

[2] K. Rummukainen, S.A. Gottlieb, Nucl. Phys. B450, 397 (1995), hep-lat/9503028

[3] C.H. Kim, C.T. Sachrajda, S.R. Sharpe, Nucl. Phys. B727, 218 (2005), hep-lat/0507006 
[4] L. Leskovec, S. Prelovsek, Phys. Rev. D85, 114507 (2012), 1202.2145

[5] R.A. Briceño, Phys. Rev. D89, 074507 (2014), 1401.3312

[6] M. Gockeler, R. Horsley, M. Lage, U.G. Meissner, P.E.L. Rakow, A. Rusetsky, G. Schierholz, J.M. Zanotti, Phys. Rev. D86, 094513 (2012), 1206. 4141

[7] R.A. Briceño, M.T. Hansen, S.R. Sharpe, Phys. Rev. D95, 074510 (2017), 1701.07465

[8] L. Alvarez-Ruso et al., Prog. Part. Nucl. Phys. 100, 1 (2018), 1706.03621

[9] L. Lellouch, M. Lüscher, Commun.Math.Phys. 219, 31 (2001)

[10] M.T. Hansen, S.R. Sharpe, Phys. Rev. D86, 016007 (2012), 1204.0826

[11] R.A. Briceño, M.T. Hansen, A. Walker-Loud, Phys. Rev. D91, 034501 (2015), 1406. 5965

[12] A. Agadjanov, V. Bernard, U.G. Meißner, A. Rusetsky, Nuclear Physics B 886, 1199 (2014)

[13] R.A. Briceño, M.T. Hansen, Phys. Rev. D94, 013008 (2016), 1509.08507

[14] R.A. Briceño, M.T. Hansen, Phys. Rev. D92, 074509 (2015), 1502.04314

[15] C. Alexandrou, J.W. Negele, M. Petschlies, A. Strelchenko, A. Tsapalis, Phys. Rev. D88, 031501 (2013), 1305.6081

[16] C. Alexandrou, J.W. Negele, M. Petschlies, A.V. Pochinsky, S.N. Syritsyn, Phys. Rev. D93, 114515 (2016), 1507.02724

[17] U.G. Meissner, J. Phys. Conf. Ser. 295, 012001 (2011), 1012.0924

[18] V. Verduci, Ph.D. thesis, Graz U. (2014), http://unipub.uni-graz.at/obvugrhs/ content/titleinfo/252105

[19] C.W. Andersen, J. Bulava, B. Hörz, C. Morningstar, Phys. Rev. D97, 014506 (2018), 1710.01557
[20] S. Paul et al., PoS LATTICE2018, 089 (2018), 1812.01059

[21] S. Durr, Z. Fodor, C. Hoelbling, S.D. Katz, S. Krieg, T. Kurth, L. Lellouch, T. Lippert, K.K. Szabo, G. Vulvert, JHEP 08, 148 (2011), 1011.2711

[22] B. Blossier, M. Della Morte, G. von Hippel, T. Mendes, R. Sommer, JHEP 04, 094 (2009), 0902.1265

[23] C. Alexandrou, L. Leskovec, S. Meinel, J. Negele, S. Paul, M. Petschlies, A. Pochinsky, G. Rendon, S. Syritsyn, Phys. Rev. D96, 034525 (2017), 1704.05439

[24] G. Rendon, L. Leskovec, S. Meinel, J. Negele, S. Paul, M. Petschlies, A. Pochinsky, G. Silvi, S. Syritsyn, PoS LATTICE2018, 073 (2019), 1811.10750

[25] S. Güsken, U. Low, K.H. Mutter, R. Sommer, A. Patel, K. Schilling, Phys. Lett. B227, 266 (1989)

[26] M. Albanese, F. Costantini, G. Fiorentini, F. Flore, M. Lombardo, R. Tripiccione, P. Bacilieri, L. Fonti, P. Giacomelli, E. Remiddi et al., Physics Letters B 192, 163 (1987)

[27] D.C. Moore, G.T. Fleming, Phys. Rev. D74, 054504 (2006), hep-lat/0607004

[28] F.A. Cotton, Chemical applications of group theory (John Wiley \& Sons, 2003)

[29] V. Bernard, M. Lage, U.G. Meissner, A. Rusetsky, JHEP 08, 024 (2008), 0806. 4495

[30] V. Pascalutsa, M. Vanderhaeghen, Phys. Rev. D73, 034003 (2006), hep-ph/0512244

[31] K. Olive, Chinese Physics C 40, 100001 (2016)

[32] C. Patrignani et al. (Particle Data Group), Chin. Phys. C40, 100001 (2016)

[33] USQCD software Qlua package, https://usqcd. lns.mit. edu/w/index.php/QLUA 